\title{
Invariantes de Curvas Planas Fechadas
}

\author{
Simone M. Moraes, Catarina M. J. Sánchez, \\ Departamento de Matemática, UFV \\ 36570-900, Viçosa, MG \\ E-mail: smoraes@ufv.br, cmendes@ufv.br,
}

\begin{abstract}
Resumo: Neste trabalho daremos uma caracterização para curvas planas, baseada nos invariantes de Arnold, e mostramos como determinar tais invariantes para curvas obtidas de cirurgias de de curvas
\end{abstract}

Palavras-chave: Curvas Planas, Invariantes de Arnold de Curvas Planas, Invariantes de Curvas, Cirurgias de Curvas

\section{Introdução}

A primeira tentativa sistemática de classificar curvas planas fechadas foi realizada por Whitney em 1937 [7]. Ele classificou as classes isotopia dessas curvas pelo índice de rotação, que é dado pelo número de voltas completas que o vetor tangente faz ao percorrer a curva considerando sua orientação.

Em 1994 V. I. Arnold publicou artigos ([2] e [3]) em que definia novos invariantes para esta classe de curvas de modo a introduzir uma nova estratificação no conjunto da curvas planas fechadas. Especificamente definiu três invariantes básicos, $J^{+}, J^{-}$e $S t$ para aplicações estáveis do círculo no plano.

Dadas duas curvas $\mathcal{C}$ e $\mathcal{C}^{\prime}$ de mesmo índice de Whitney existe uma família $\mathcal{F}$ a 1-parâmetro de imersões de $S^{1}$ em $\mathbb{R}^{2}$ conectando $\mathcal{C}$ e $\mathcal{C}^{\prime}$, as imersões conectando essas curvas podem ser não-genéricas. No caso em que a aplicação que define a família $\mathcal{F}$ é genérica, de Arnold [2], podem ocorrer três tipos de eventos: auto-tangência direta, auto-tangência inversa e pontos triplos.

Arnold introduziu três invariantes de curvas plans fechadas não-genéricas, na mesma classe de imersão, da seguinte forma:

$J^{+}$associado às tangências diretas (com orientações concordantes).

$J^{-}$associado às tangências inversas (com orientações opostas)

St associado aos pontos triplos.

Estes invariantes estimam o número de cruzamentos ao longo de um caminho, no espaço das aplicações suaves, que leva uma curva genérica em outra. Arnold mostrou que estes invariantes são aditivos em relação a soma conexa de curvas.

Em [6] foram introduzidos canais e pontes de curvas planas, que facilitam o cálculo destes invariantes. Uma ponte consiste em introduzir um retângulo no complemento da curva no plano, respeitando as orientações, decompondo a curva dada em duas curvas menores com invariantes conhecidos. O invariante da curva pode ser obtido em função dos invariantes das duas curvas e do índice da ponte em relação a curva dada.

Neste trabalho, introduziremos a caracterização de curvas planas e apresentaremos um generalização de pontes e canais para curvas planas, introduzida em [4], com todos os possíveis canais e pontes, que permite calcular os invariantes de Arnold para uma curva plana em função 
das pontes e de curvas com no máximo um ponto duplo. O principal resultado é o seguinte teorema:

Teorema: Uma curva com $n$ pontos duplos é uma (curva) árvore se, e somente se, admite uma decomposição de exatamente $n$ curvas dos tipos $K_{0}$ e $K_{2}$ e pontes sem pontos duplos ou uma decomposição de exatamente $n+1$ curvas do tipo $K_{1}$ (isotópicas ao círculo) com pontes de pontos duplos.

Como corolário deste resultado obtemos as fórmulas para as famílias de curvas árvores, proposto como conjectura por Arnold em [2] e provado por Aicardi em [1].

\section{Invariantes de Arnold de Curvas Planas}

\subsection{Preliminares}

Os conceitos básicos de curvas planas serão introduzidos a continuação.

Definição 2.1. Uma curva plana fechada suave é uma aplicação diferenciável do círculo $S^{1}$ no plano, cujas derivadas de todas as ordens nunca se anula. Vamos designa-lá simplesmente por curva plana.

Definição 2.2. Um ponto duplo em uma curva plana é a imagem de dois pontos distintos do círculo com a propriedade de que os vetores tangentes são distintos.

Definição 2.3. O indice de uma curva plana fechada é o número de rotações do vetor tangente à curva.

Teorema 2.4. (Teorema de Whitney [7]) Uma curva plana fechada $\mathcal{C}$ pode ser deformada em um curva plana fechada $\mathcal{C}^{\prime}$ se, e somente se, $\mathcal{C}$ e $\mathcal{C}^{\prime}$ têm o mesmo indice.

\subsection{Os Invariantes $J^{+}, J^{-}$e $S t$}

Em [2] Arnold mostrou que somente pontos triplos e auto-tangências são necessários para moverse no espaço das curvas genéricas. Estes pontos especiais são passados através dos seguintes movimentos:

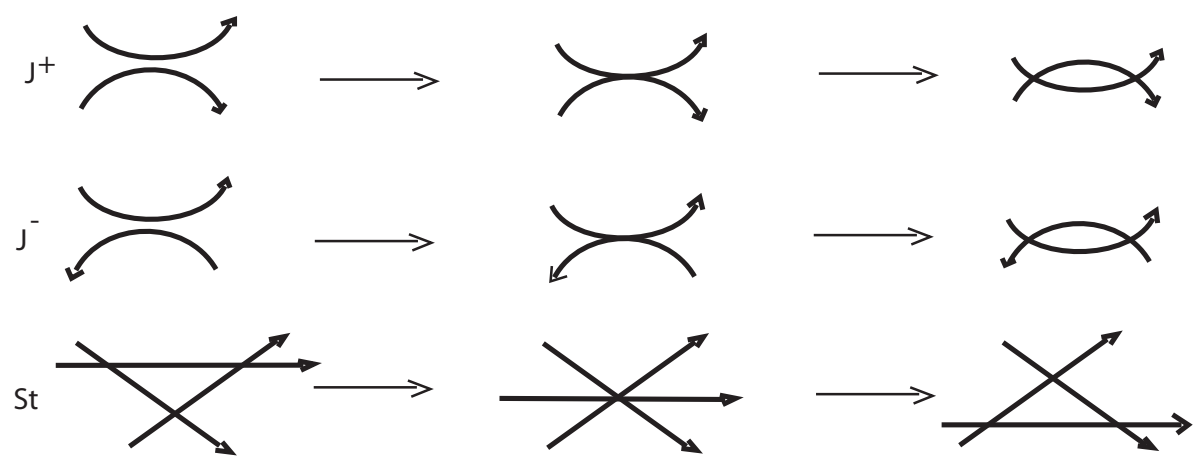

Figura 1: Movimentos básicos

Observação 2.5. Um movimento $J^{ \pm}$é positivo se o número de pontos em dobro aumenta, e negativo se este número diminui. Um movimento St é positivo se o triângulo que surge é orientado positivamente, e negativo se este triângulo é orientado negativamente.

Definição 2.6. Um triângulo desaparecendo/surgindo é um triângulo formado por três ramos de uma curva antes/depois de um movimento St. 


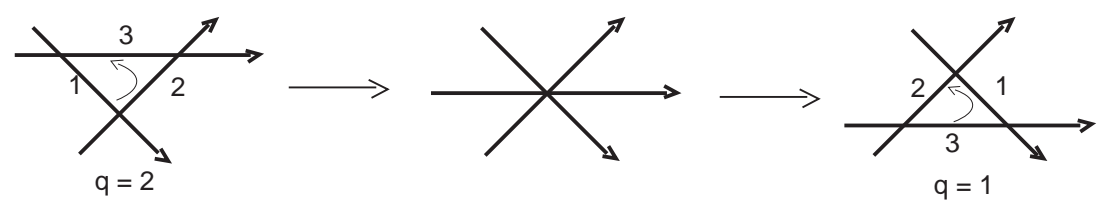

Figura 2: Regra do Triângulo.

O sinal de tal triângulo é dado por $(-1)^{q}$, com $q$ o número de lados do triângulo que coincide com a orientação cíclica dada pela orientação da curva.

Para definir os valores dos invariantes $J^{+}, J^{-}$e $S t$ Arnold considerou uma família de curvas básicas ilustradas na figura 3, que vamos designar por curvas básicas de Arnold, de fato toda curva fechada regular pode ser deformada em uma curva básica da Arnold.

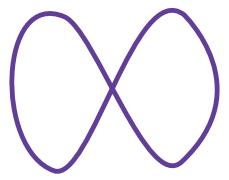

$\mathrm{K}_{0}$

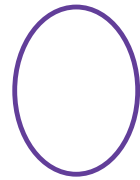

$\mathrm{K}_{1}$

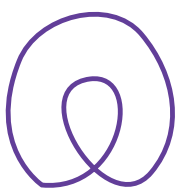

$\mathrm{K}_{2}$

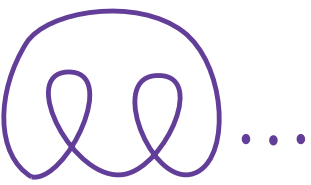

$\mathrm{K}_{3}$

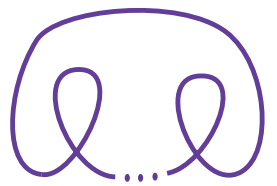

$\mathrm{K}_{\mathrm{n}}$

Figura 3: Família de Curvas Básicas.

Arnold mostrou ainda que esses invariantes estão bem definidos e são únicos a menos de uma constante e associou às curvas básicas os seguintes valores:

$$
\begin{array}{lll}
S t\left(K_{0}\right)=0 & S t\left(K_{i+1}\right)=i & \\
J^{+}\left(K_{0}\right)=0 & J^{+}\left(K_{i+1}\right)=-2 i & \text { para } i=0,1,2,3, \ldots \\
J^{-}\left(K_{0}\right)=-1 & J^{-}\left(K_{i+1}\right)=-3 i &
\end{array}
$$

Definição 2.7. A soma conexa disjunta de duas curvas, cada uma em semi-planos distintos, é obtida ao conectar as duas imersões originais através de dois arcos paralelos, mergulhados ("ponte"), interceptando apenas nas extremidades. Como mostra a figura 4.

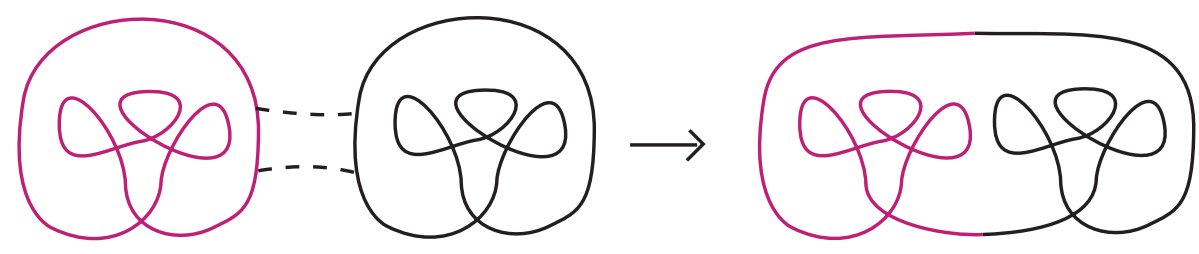

Figura 4: Soma Conexa de Curvas Planas.

Teorema 2.8. Todos os invariantes básicos são aditivos sob a soma conexa de imersões.

Observação 2.9. As somas conexas das curvas básicas satisfazem:

(a) $K_{i+1}=\underbrace{K_{2}+\cdots+\cdots+K_{2}}_{i \text { vezes }}=i K_{2}$,

(b) $K_{i+1}+K_{j+1}=K_{(i+j)+1}, i>0$ e $j>0$.

Logo, $X\left(K_{j+1}+K_{l+1}\right)=X\left(K_{j+l+1}\right)=X\left(K_{j+1}\right)+X\left(K_{l+1}\right)$, para $X=J^{+}, J^{-}$, St.

Definição 2.10. Dizemos curva arborescente ou do tipo árvore se a eliminação de qualquer um de seus pontos duplos decompõe esta em duas curvas disjuntas (veja exemplo na figura 5).

Dizemos que uma curva $\Gamma$ com $n$ pontos duplos é extrema se $i(\Gamma)=n+1$ (uma curva extrema é arborescente, veja exemplo na figura 5b). 
a)

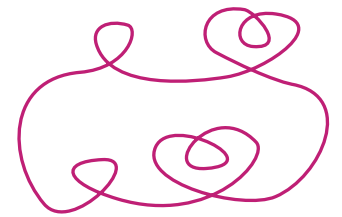

b)

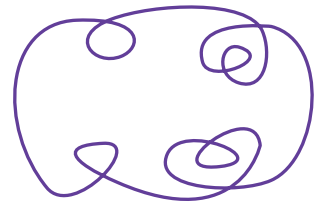

Figura 5: Curvas Arborescentes.

\section{Invariantes de Famílias de Curvas Planas Fechadas}

Uma consequência importante deste estudo é a obtenção de um método para calcular os invariantes de Arnold, baseado na decomposição de uma curva dada em curvas básicas unidas por pontes. Obtemos assim uma fórmula que proporciona os invariantes da curva inicial em termos dos invariantes das curvas básicas que aparecem na decomposição. Veremos com alguns exemplos que este método reduz consideravelmente com relação a outros métodos já conhecidos (veja, por exemplo (Polyak e Viro [5]).

Além disso, estendemos o estudo de Arnold a coleções de curvas determinando em particular invariantes $T^{+}\left(\Gamma_{1}, \Gamma_{2}\right), T^{-}\left(\Gamma_{1}, \Gamma_{2}\right)$ e $T^{S t}\left(\Gamma_{1}, \Gamma_{2}\right)$, para pares de curvas planas que são relevantes no estudo das aplicações estáveis das superfícies no plano. E obtemos, diretamente, os resultados de Aicardi [1] para curvas arborescentes.

\section{Notações:}

- $\vartheta_{\gamma}= \pm 1$, de acordo com as orientações dos arcos conectados por $\gamma$.

- $\eta_{\gamma}$ indica o número de componentes conexas que $\gamma$ conecta

- $i\left(\gamma^{ \pm}\right)=\mp 1$ e $i\left(\gamma^{0}\right)=0$.

Definição 3.1. $T^{+}\left(\Gamma_{1}, \Gamma_{2}\right)$ é o número total de tangências diretas, com seus sinais contados, formadas ao longo do processo de separação de duas curvas e $T^{-}\left(\Gamma_{1}, \Gamma_{2}\right)$ é o número de tangências inversas, com seus respectivos sinais, que aparecem neste processo.

Seja $P$ um ponto triplo formado ao longo da separação destas duas curvas, denotamos por $\xi_{p, q}(P)$ o sinal correspondente ao ponto triplo $P$ que pode tomar valores +1 ou -1 de acordo com o seguinte critério:

- Percorrendo estas curvas a partir dos pontos base respeitando a ordem e orientação das mesmas, isto determina uma ordem cíclica nos três arcos que formam um dado ponto triplo, assim, o sinal desta transição é determinado pela regra do triângulo.

Definição 3.2. O número $T^{S t}$ de duas curvas $\Gamma_{1}$ e $\Gamma_{2}$, denotado por $T^{S t}\left(\Gamma_{1}, \Gamma_{2}\right)$ é dado pela soma $\sum_{P} \xi_{p, q}(P)$, esta soma tem contribuições de todos os pontos triplos $P$ que são formados pelo par de curvas, ao longo do processo de separar as curvas em semi-planos disjuntos.

\subsection{Pontes}

Sejam $\Gamma$ um conjunto de curvas planas e $\gamma$ uma outra curva contida no seu complemento, conectando dois $\operatorname{arcos} A, B \subset \Gamma$, entre os respectivos pares de pontos de intersecção $\left(p_{1}, q_{1}\right)$ e $\left(p_{2}, q_{2}\right)$, da seguinte forma:

Substituímos os arcos $x_{1} y_{1} \subset A$ e $x_{2} y_{2} \subset B$, pelos arcos $\bar{x}_{1} \bar{y}_{2}$ e $\bar{x}_{2} \bar{y}_{1}$ da curva $\gamma$, de tal forma que os arcos $p_{1} \bar{x}_{1} \bar{y}_{2} q_{2}$ e $p_{2} \bar{x}_{2} \bar{y}_{1} q_{1}$ respeitam as respectivas orientações das curvas em $\Gamma$ e sejam suaves.

A conexão de $\gamma$ no conjunto de curva $\Gamma$, denotada por $\gamma * \Gamma$, é compatível se os arcos $A$ e $B$ com orientação de $\Gamma$ definem um orientação em $\gamma$, como mostra a figura 6 . 
Definição 3.3. Dizemos que $\gamma$ é uma cirurgia em $\Gamma$ se $\gamma$ é uma uma curva do tipo "O" ou " $\infty$ " (veja figura 6).
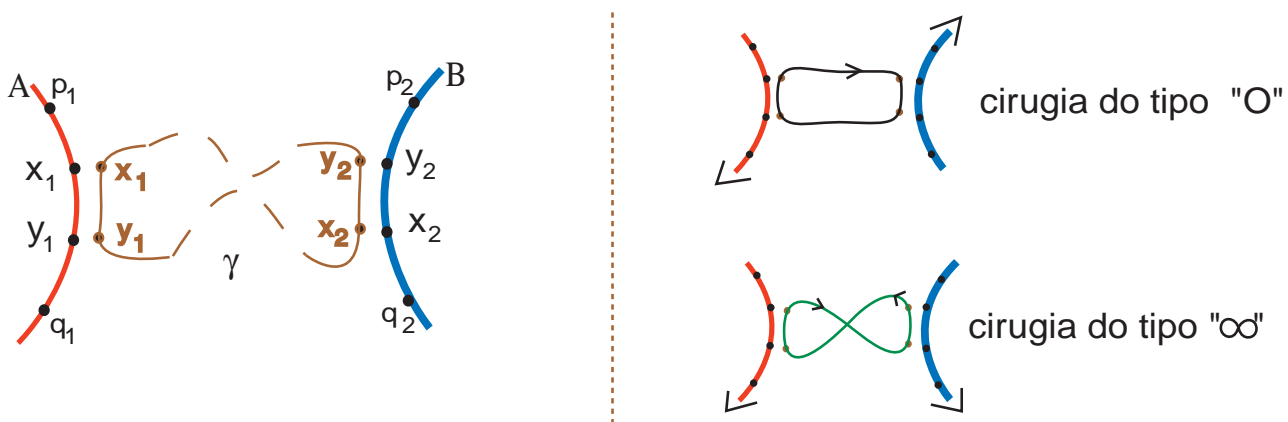

Figura 6: Cirurgia nos arcos de $\Gamma$.

Definição 3.4. Dada um conjunto de curvas $\Gamma$, seja $\gamma$ uma curva conexão simples (do tipo "O" ou " $\infty$ ") em $\Gamma$. Dizemos que $\gamma$ é uma ponte.

No caso em que $\gamma$ conecta arcos de uma mesma componente conexa também dizemos que $\gamma$ é um canal.

Observação 3.5. 1. Se $\gamma$ conecta arcos de componentes conexas distintas, então $\eta_{\gamma}=0$.

2. Se $\gamma$ conecta arcos de uma mesma componente conexa, então $\eta_{\gamma}=1$.

Denotaremos, quando necessário, $\gamma^{0}$ quando a cirurgia é do tipo se " $\infty$ " $\left(\vartheta_{\gamma}^{0}=0\right)$ e por $\gamma^{ \pm}$ quando a cirurgia é do tipo " $O$ " e $\vartheta_{\gamma}= \pm 2$.

$\mathrm{Na}$ vizinhança de um ponto de intersecção temos quatro possibilidades diferentes de conectar dois arcos, como mostra a figura 7a). Observe que uma cirurgia tipo " $O$ " sempre conecta regiões do complemento $\mathbb{R}^{2} \backslash \Gamma$ de índices iguais.

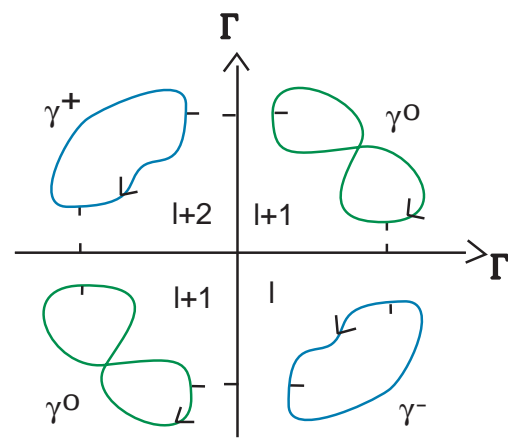

a)

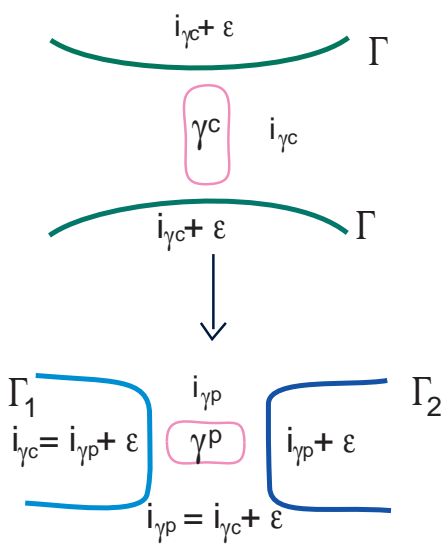

b)

Figura 7: Pontes

Exemplo 3.6. A figura 8 ilustra um exemplo com $\Gamma=\left(\Gamma_{1}, \Gamma_{2}\right)$, com $i\left(\Gamma_{1}\right)=4$ e $i\left(\Gamma_{2}\right)=1$. Os indices destas componentes de $\Gamma$ conectadas pelas diferentes pontes são:

$$
i\left(\gamma_{1}^{-} * \Gamma\right)=i\left(\gamma_{2}^{-} * \Gamma\right)=4, i\left(\gamma_{3}^{+} * \Gamma\right)=6 \text { e } i\left(\gamma_{4}^{0} * \Gamma\right)=5 .
$$




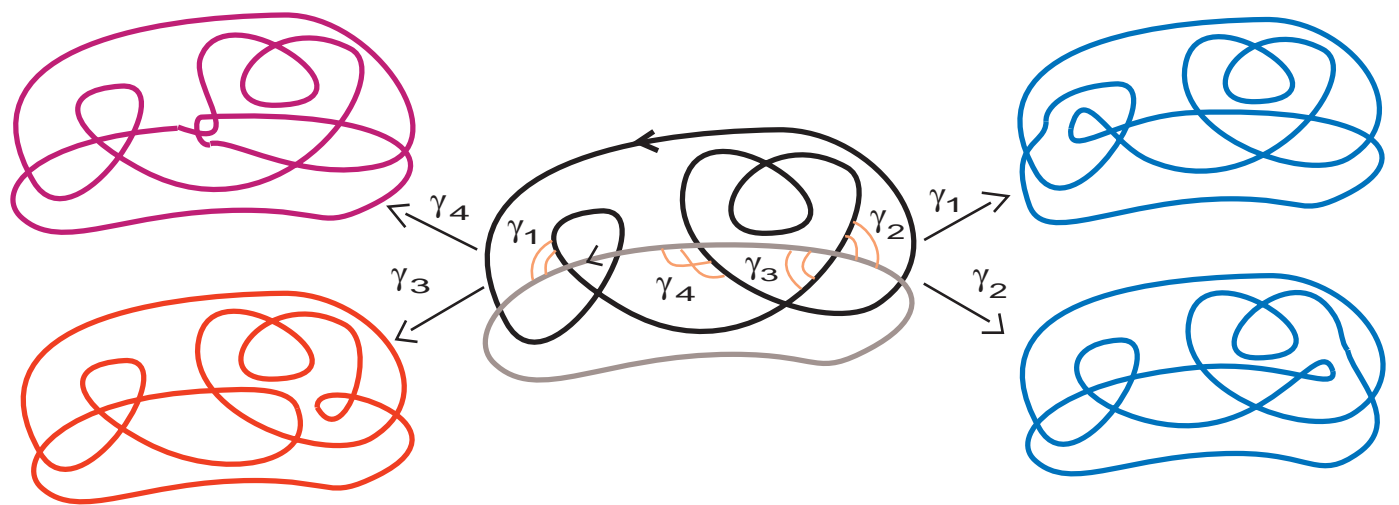

Figura 8: Soma de duas curvas com diferentes pontes.

\subsection{Os invariantes $J^{+}, J^{-}$e $S t$ para $\gamma * \Gamma$}

Vamos determinar o efeito da cirurgia $\gamma$ (cirurgias) sobre os invariantes de Arnold.

Os principais resultados demonstrados são:

Teorema 3.7. Sejam $\Gamma$ um conjunto de curvas e $\gamma$ uma ponte conectando dois arcos $A$ e $B$ de $\Gamma$, então

$$
J^{ \pm}(\gamma * \Gamma)=J^{ \pm}(\Gamma)+J^{ \pm}(\gamma)-\left(\vartheta_{\gamma} i_{\gamma}-2 \eta_{\gamma}\right) .
$$

Observação 3.8. No caso em que $\Gamma$ tem apenas duas componentes de curvas separadas em semi-planos disjuntos, isto é, $\eta_{\gamma}=i_{\gamma}=0$, temos

$$
J^{ \pm}(\gamma * \Gamma)=J^{ \pm}(\Gamma)+J^{ \pm}(\gamma)=J^{ \pm}\left(\Gamma_{1}\right)+J^{ \pm}\left(\Gamma_{2}\right)+J^{ \pm}(\gamma),
$$

resultado foi demonstrado por Arnold em [3].

Teorema 3.9. Sejam $\Gamma_{1}$ e $\Gamma_{2}$ duas componentes de curvas conectadas por uma ponte $\gamma$, então

$$
S t\left(\gamma *\left(\Gamma_{1}, \Gamma_{2}\right)\right)=S t\left(\Gamma_{1}\right)+S t\left(\Gamma_{2}\right)-T_{\gamma}^{S t}\left(\Gamma_{1}, \Gamma_{2}\right) .
$$

\subsection{Invariantes para Curvas Arborescentes}

Em [1], Aicardi demonstra fórmulas, conjecturadas por por Arnold em [2], para várias curvas arborescentes (tipo árvore) especiais. Aqui obteremos uma destas fórmulas como consequência direta de nossos resultados.

O primeiro mostramos uma equivalência entre uma curva ser do tipo árvore e sua decomposição em curvas do tipo $K_{0}, K_{1}$ e $K_{2}$.

Teorema 3.10. Seja $\Gamma$ uma curva com $n$ pontos duplos. $\Gamma$ é uma curva do tipo árvore se, e somente se, admite uma decomposição de exatamente $n$ curvas dos tipos $K_{0}$ e $K_{2}$ e pontes dos tipos $\gamma^{ \pm}$ou uma decomposição de exatamente $n+1$ curvas do tipo $K_{1}$ (isotópicas ao círculo).

Corolário 3.11. Seja $\Gamma$ uma curva árvore com $n+1$ pontos duplos e $\gamma * \Gamma$ uma decomposição de $\Gamma$. A curva $\Gamma$ é extrema se, e somente se, cada componente de $\gamma * \Gamma$ é do tipo $K_{2}$ e cada componente de $\gamma$ é do tipo $\gamma^{+}$.

Corolário 3.12. Os invariantes de uma curva extrema $\Gamma$ com $n$ pontos duplos são dados por:

(i) $J^{+}(\Gamma)=-2 \sum_{i=0}^{n} i_{p_{i}}$,

(ii) $J^{-}(\Gamma)=-n-2 \sum_{i=0}^{n} i_{p_{i}}$, 
(iii) $\operatorname{St}(\Gamma)=\sum_{i=0}^{n} i_{p_{i}}$

com $p_{i}$ os pontos duplos da curva para $i=1, \cdots, n$, .

Exemplo 3.13. [1] Considere a família de curvas extremas, $C_{n}$, dado na figura 9. Observe

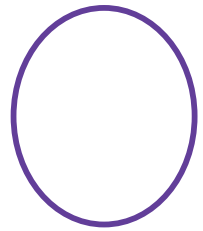

$\mathrm{C}_{0}$

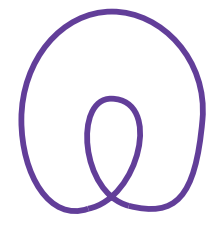

$\mathrm{C}_{1}$

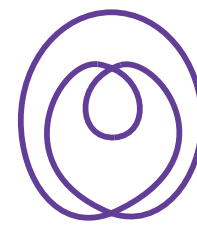

$\mathrm{C}_{2}$

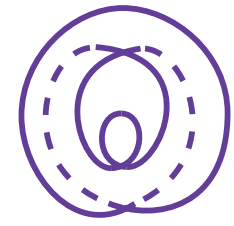

$\mathrm{C}_{\mathrm{n}}$

Figura 9: Família $\left\{C_{n}\right\}_{n \geq 0}$.

que se enumeramos os pontos duplos de fora para dentro da curva como $p_{1}, \cdots, p_{n}$ temos que $i_{p_{j}}=j$. Segue do corolário 3.12 que:

$$
\left\{\begin{array}{l}
S t\left(C_{n}\right)=\frac{n^{2}+n}{2} \\
J^{-}\left(C_{n}\right)=-\left(n^{2}+2 n\right) \\
J^{+}\left(C_{n}\right)=-\left(n^{2}+n\right)
\end{array}\right.
$$

\section{Referências}

[1] F. Aicardi, Tree-like Curves. In: Singularities and Bifurcations. Advances in Soviet Mathematics, 21, AMS, Providence, 1-36, 1994.

[2] V. I. Arnold, Topological Invariants of Plane Curves and Caustics. University lecture series, Vol. 5, AMS Providence, 1994.

[3] V. I. Arnold, Plane Curves, Their Invariants, Perestroikas and Classificacions. Advances in Soviet Mathematics, Vol. 21, 1994. American Mathematical Society, 1994.

[4] C. Mendes de Jesus, Invariantes Topológicos de Aplicações Genéricas de Superfécies Compactas Orientáveis no Plano, Tese de Doutorado, PUC-RIO, 2001.

[5] M. Polyak e O. Viro, Invariants of Curves and Fronts via Gauss Diagrams, International Mathematics Research Notices, No 11, 1994.

[6] C. Mendes de Jesus e M. C. Romero Fuster, Bridges, channels and Arnold's invariants for generic plane curves, Topology and its Applications 125, 505-524, 2002.

[7] H. Whitney, On regular closed curves in the plane. Compositio Math., 4 pags. 276-284, 1937. 